\title{
A Tool Development for Test Case Based Code Optimization in Java
}

\author{
Turgay Taymaz ${ }^{1 *}$ (D), Kökten Ulaş Birant ${ }^{2}$
}

\begin{abstract}
Java has been a popular programming language since its first stable release in 1996 because of its platform independence. Along with its popularity Java has been a focus of performance studies since its debut. Developments in hardware has unbelievably advanced the performance of the devices that run Java and thus software performance has lost its popularity until the release of Android OS and rapid increase in mobile device ownership Java language usage has increased once again. Mobile devices having far less system resources compared to personal computers had re-brought software performance studies into the spotlight. However mobile devices have gone into a fast-paced development like all other information technologies and this brought down the need for software performance studies, again. Also, worth mentioning that development of new Java Virtual Machine (JVM) versions has made the specialized compiler studies, which may threaten the platform independency, obsolete except for specific situations. Today it is not enough to consider code optimization solely in terms of performance improvement. Much broader vision is needed like software development processes such as Maintainability, code readability, improving cooperation in multi-programmer projects, software quality assurance.
\end{abstract}

In this study, white box testing approach is adopted as the software testing technique and static code analysis method is selected to ensure line coverage. A new software (JPA) has been developed based on a currently used testing tool (PMD) to improve the user experience.

Keywords: Java, code optimization, PMD, static code analysis.

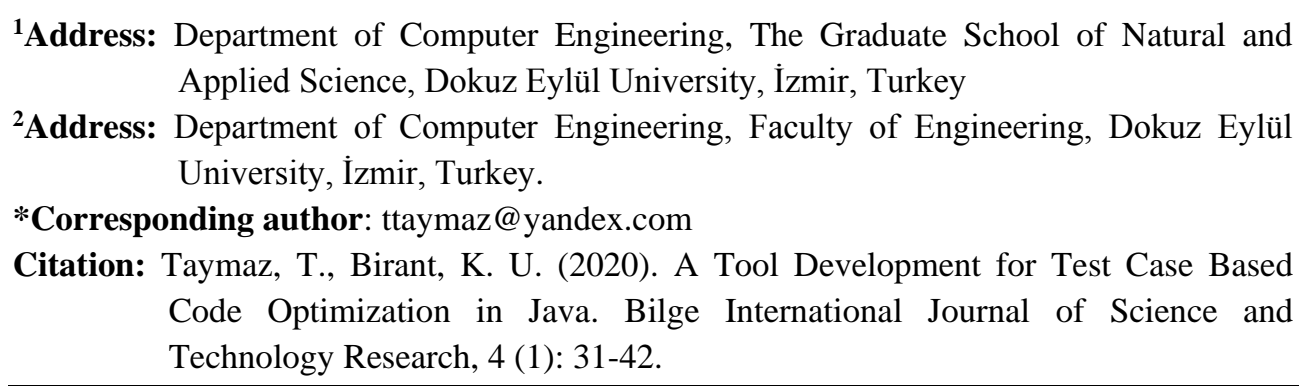

\section{INTRODUCTION}

Rose flower is seen as a symbol of purity, beauty, love and Java programming language is a general-purpose, concurrent, class based, object-oriented language which allows application developers to write programs that can run on any platform; either online or on different types of devices (Gosling et al., 2018). Java compiler converts the source code to bytecode, which then can be executed on any operating system using JVM (Java Virtual Machine). Independence from operating systems provides flexibility and simplicity, which has considerably increased the popularity to this programming language since the release of version 1.0 in 1996. However, being flexible can also lead the developers to a non-focused implementation and in turn might make some applications to be less efficient than other platform dependent programming languages, which brings out a need for performance improvement applications for Java.

While performance studies have been conducted, starting with the first version of Java programming language with projects such as High Performance Java (HPJAVA) and The Ninja Project, these studies have discontinued due to Java Development Kit (JDK) updates and due to the fact that developments in computer hardware have rendered these studies redundant. (Carpenter et al.,1997; Moreira et al., 2001). Nevertheless, performance studies for Java came back into focus again with the announcement of the Android operating system in 2008.

Android operating system is based on an open-source distribution of Linux operating system. Programmers can 
develop Java-based applications and deploy them on Android devices (Hall \& Anderson 2009). There has been a steady growth from the start in the numbers of the applications that were developed for Android OS because Java was already a popular and well-known programming language when Android OS was commercially released in 2008. Today, Android OS is the most popular operating system for all devices surpassing even Microsoft Windows, given in Figure 1.

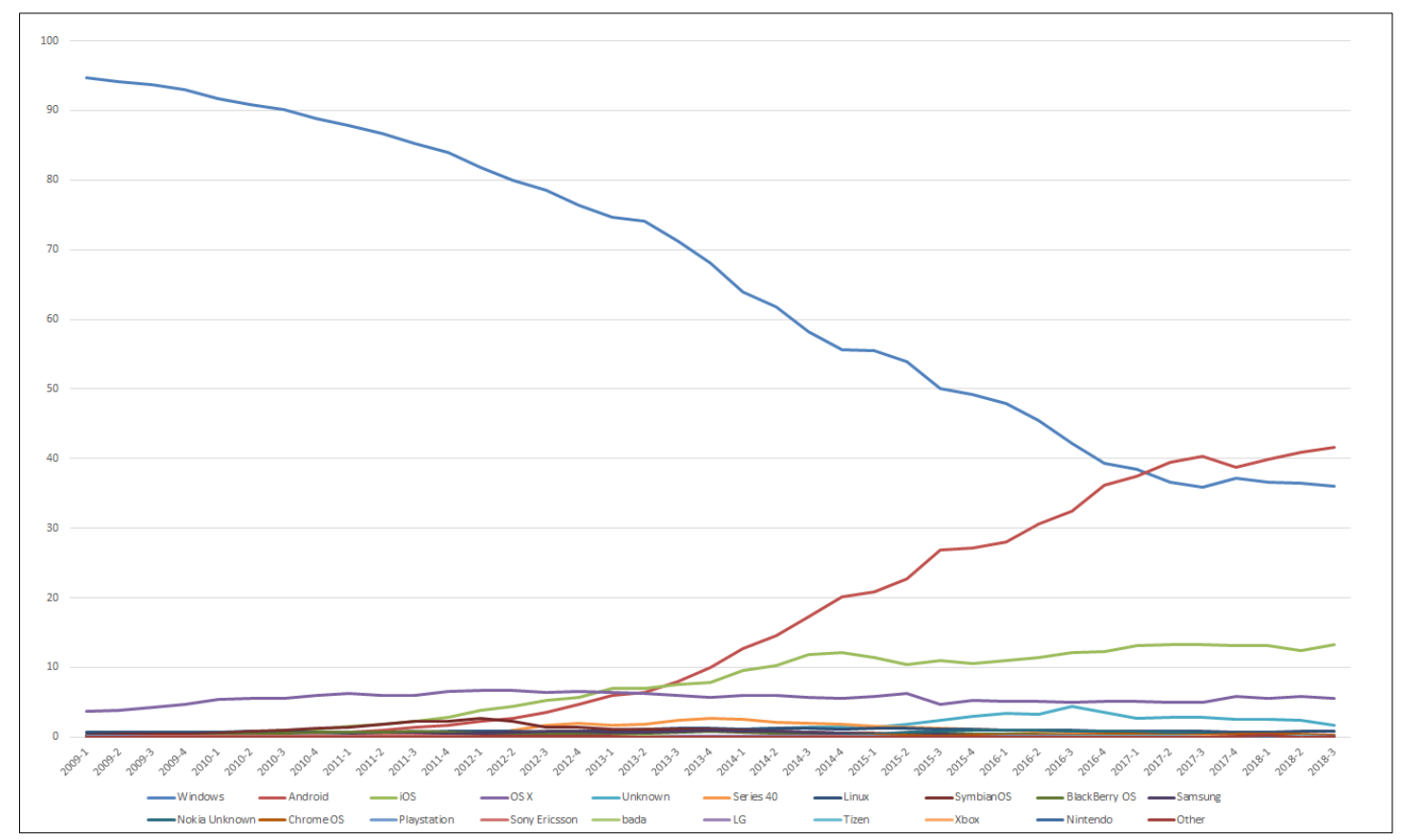

Figure 1. Operating system market share worldwide (Statcounter, n.d.)

\section{Material and Method}

\subsection{What is code optimization}

A definition that most programmers will agree is that the main purpose of code optimization is to increase the quality of the code in terms of time and space without affecting the output result of the code (Bajwa et al., 2016).

The terms time and space lead to a common perception of code optimization as only the performance increase; in a wider perspective, optimization may also mean an improvement in other aspects as defined below.

Code optimization is the process of modifying source code to improve code quality and efficiency. For instance, a program can be optimized so that it runs faster, or works with less memory storage or other resources, or consumes less power, or can be more readable to facilitate maintenance and updating (Bajwa et al., 2016; Johnson 2008; Lins, 2017; Palaniappan, 2016).

Code optimization can be performed at levels such as design level, algorithms and data structures, source code level, build level, compile level, assembly level and run time (Lins, 2017). In general, higher optimization levels have a greater impact and are more difficult to change later in a project, so they require a complete rewrite if they need to be changed. For this reason, optimization generally proceeds from higher to lower levels. Larger gains can be achieved at higher levels with less effort, then gains get smaller and require more effort as levels go lower. However, this is not always the case, in some cases the performance of the program may show tremendous increases in performance with small changes made at lower levels. Therefore, it is not possible to foresee whether the time and effort required are worth the performance increase, not to mention the unforeseen errors that may occur. Because of this unpredictability, the changes made for optimization can be abandoned, partially abandoned or postponed to a later date, depending on the size and complexity of the project.

Assuming the code optimization only as performance enhancements would compromise the stability of the program. Because such an approach would be ignoring the concept of code quality as in debugging, maintenance efforts and design process of later versions.

\subsection{When to optimize?}

Choosing the right programming language and platform in the design phase will be the most basic start for optimization. The right architecture selection is also made at this beginning stage since it can be very challenging to change later. In general, it will be even more difficult to change the data structure than the algorithm because the data structure assumption and its performance assumption will be used throughout the entire program. For the sake of improving performance, adding new codes and changing 
source code may reduce readability. This can result in serious complications in maintaining and debugging the program. Therefore, optimization for performance improvement is better to be left to the end of the development phase.

Premature optimization is the (so-called) improvement effort in an immature system. The following quote is about premature optimization from Donald Knuth: "The real problem is that programmers have spent far too much time worrying about efficiency in the wrong places and at the wrong times; premature optimization is the root of all evil (or at least most of it) in programming."(Knuth, 1974).

Indisputably that was a different time when mainframes and punch cards were common and CPU processing cycles were also scarce. With advancing technology, innovations such as much higher CPU processing cycles have emerged, still premature optimization has become a controversial issue.

Nowadays, programs can be quickly distributed over the Internet and the codes are updated if necessary, afterwards. A classic example of this would be a start-up that spends a lot of time trying to figure out how to scale their software to handle millions of users. This may seem like a valid concern to be considered. But it makes more sense to worry about processing millions of users, after making sure that at least 100 users like this product and want to use it. If the product is coded in an easy to maintain, the necessary optimizations are easily taken care of (Watson, 2017).

Developments in compilers made some optimizing operations unnecessary, such as bitwise shift and mask used to divide or multiply a positive integer expression by two, because the compiler performs these operations automatically when compiling the code. As a result, ease of maintenance by writing readable code has come to the fore once again.

Before starting optimization, it would be more useful to prepare a report of the program code including suggestions in source code level and then apply the selected suggestions and re-report including a comparison between the multiple versions previously tested by the user.

\subsection{Optimizing Java source code}

In order to understand Java code optimization, it is necessary to explain the technology behind the Java programming language and the development of this technology. In other programming languages the compiler generates machine code for a particular system. But in the Java programming language, the compiler generates its own alternative format, which is called bytecode, for Java Virtual Machine (JVM) instead of the machine language. JVM is an abstract computing machine and provides Java programming language hardware and platform independence.

Just-In-Time (JIT) compiler improves the performance of Java applications at run time and it is a component of JVM.
JVM loads the class files at program runs time. The class files determine the meaning of each bytecode and make the appropriate calculation. For comparing to a native application, additional processor and memory usage during interpretation may cost Java application extra time. However, as JIT completes the compilation, the application reaches its peak performance approaches the performance of a native application.

In Java programming language, to make performance improvements, codes can be refactored, or settings can be adjusted on the compiler, and even a new compiler can be designed to generate bytecode for JVM. However, in this study, instead of these options, it was aimed to create a report by examining the source code with static code analysis and to increase the optimization by increasing the code quality depending on this report. The main reasons for this are listed below.

Java is a language licensed by General Public License developed by Oracle and is regularly updated. With these updates, there is also an increase in performance.

The execution speed of Java code is highly dynamic and fundamentally depends on the underlying Java Virtual Machine. An old piece of Java code may well execute faster on a more recent JVM, even without recompiling Java source code (Evans et al., 2018). Combining this fact with the possibility that refactoring may not sufficiently improve the software performance, refactoring approach was not pursued in this research. Although there may be optimizations that can still be applied for special circumstances and unforeseen cases in the future, they cannot be generalized and may not be expected for the same performance increase in the upcoming Java versions.

JDK compilers can be customized to create a more efficient bytecode for JVM. Additionally, new programming languages such as Scala and Kotlin have been developed and are available as alternatives, which can also work with JVM. Scala combines object-oriented and functional programming in one concise, high-level language. Scala's static types help avoid bugs in complex applications, and its JVM and JavaScript runtimes let you build highperformance systems with easy access to huge ecosystems of libraries (Scala n.d.). Kotlin is a statically typed programming language that runs on Java Virtual Machine and can also be compiled to JavaScript source code or use LLVM compiler infrastructure (Kotlin n.d.).

Considering the above-mentioned information, working to optimize the software at the source code level appears much more promising than just increasing the performance for current runtime version. Additionally, leaving the final decision to the programmer by producing reports provides flexibility for the programmer who may have specific needs or specific requirements. For this purpose, statement coverage, a White-Box Test technique, is implemented in this study. 


\subsection{Software testing}

Software testing is used to expose defects and errors in the software. Principal benefits that can be gained by testing are software quality assurance, reliability estimation of software, validation and verification. Software testing is a key component of software quality assurance and represents a refinement of specification, design and coding (Khan \& Khan, 2012).

The primary purpose of the software test is to verify the quality of the software system, another purpose is to determine the integrity and accuracy of the software and ultimately reveal undiscovered errors. Software testing ensures an effective performance of the application (Singh \& Kazi, 2016).

Software can be tested with box approach. There are essentially two types of the "box" approach: black box testing and white box testing. The combination of said approaches is called grey box testing. Figure 2. shows the "box" approaches in software testing.

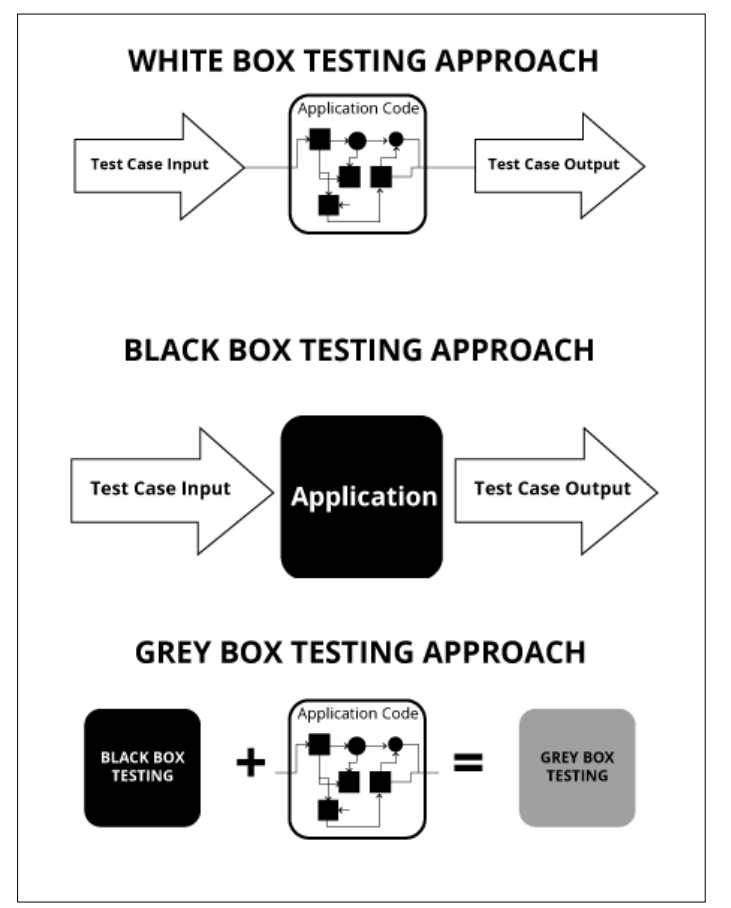

Figure 2. Software testing "Box" approaches (Invensis, n.d.)

White box testing deals entirely with the code structure. Both the source code and the compiled code of the project are tested. Unlike white box testing, black box approach, allows testing to be performed without any requirement of inside knowledge of the code structure or design of the project. The comparison of the input and output can obviously only test if the functional requirements of the system are met or not. Grey box testing, as previously stated, is a combination of white-box testing and black-box testing approaches. Although Grey box testing depend on some inside knowledge of the code structure, it is actually platform and language independent. The reason behind this is; it is essentially a black box test modified according to the main data structures and algorithm of the application but not the details of the code. (Sawant et al., 2012; Jovanović, 2009).

Black box testing doesn't need any knowledge of the internal structure or coding in the program, and more importantly doesn't give any conclusions or suggestions about it either. Similarly, grey box testing doesn't require that the tester have access to the entire source code and again limited to boundary values and interfaces between program modules. White box testing is preferred in this study because it is focused on the code and would give more meaningful results pertaining to the code structures (Jovanović, 2009).

\subsubsection{White box testing}

White box testing is sometimes referred to as clear box testing, glass box testing, transparent box testing due to its access to the codes and algorithms or structural testing because of its focus on internal structure or working of a software, rather than its functionality (Karnavel \& Santhoshkumar, 2013).

Performing white box testing technique follows a step by step approach and tries to verify each program statement even the comments. White box testing enables performance of data processing and calculations correctness tests, software qualification tests, maintainability tests and reusability tests. The implementation of white box testing is based on controlling the data processing for each test case which in turn raises the issue of coverage of a huge number of possible processing paths and the numerous lines of code. This adversity has given rise to two approaches called "Path coverage" and "Statement coverage". Path coverage is to check whether all possible routes are applied along a certain part of the code. Statement coverage, also known as line coverage, is verifying whether each statement in the program is executed or not (Galin, 2004).

In a software structure, different paths are created by conditional expressions such as IF - ELSE, DO - WHILE. Path testing attempts to provide the full scope analysis of a program by testing all possible paths. Therefore, "path test's completeness" is defined as the percentage of program paths carried out during the test. The concept of path testing is not practical in most cases because of the vast resources needed for its performance. Because of this predicament, statement coverage has been developed as an alternative. In statement coverage, test cases leave most of the possible paths untested however requires far fewer test cases to cover all paths compared to path coverage. As an alternative to creating multiple test cases to cover all paths, static code analysis is a viable solution, or even better, using a tool to automate static code analysis (Galin, 2004).

\subsection{Static code analysis}

Static code analysis, also called static analysis, is the method of examining the program codes without the actual execution. Static analysis can be considered a code review process. Code reviewing is one of the oldest and safest 
methods for detecting errors in the source. It suggests reading the source code carefully and make suggestions on how to improve it. This process is used to locate existing errors and pieces of code that may cause future errors. Code review process is useful, because programmers are more easily to notice others' mistakes than their own. The most important problem in this process is the need for periodical meetings of programmers to review each new code, or rereview a code after the proposed changes are applied. When programmers review large pieces of code at a time, they lose their attention quickly, so they need to rest regularly. Otherwise, code review will not help. This is a serious problem because of its immense cost in man hours. Automation of static analysis, i.e. static code analysis tools would be a good solution to reduce this cost (Ayewah et al., 2008).

\subsubsection{Static code analysis tools}

Static code analysis tools examine the source code of programs and give suggestions to the programmer as to which parts of the code to reconsider. These tools may not replace a code review by a team of programmers, but the benefit/cost ratio makes the use of static analysis a very good option. Static code analysis tools are very successful in detecting errors in programs and providing code formatting suggestions. One of the main advantages of static analysis is that it allows the cost of eliminating errors in the software to be greatly reduced. This is mainly because this analysis can be performed at the coding stage. Fixing an error at the testing stage costs about ten times more compared to development stages, as shown in Table 1. Static analysis can be performed in construction, system test and post-release phases.

Table 1. Average cost of fixing defects based on when they're introduced and detected (McConnell, 2014)

\begin{tabular}{|l|c|c|c|c|c|}
\hline & \multicolumn{5}{|c|}{ Time Detected } \\
\hline Time Introduced & Requirements & Architecture & Construction & System Test & Post-Release \\
\hline Requirements & 1 & 3 & $5-10$ & 10 & $10-100$ \\
\hline Architecture & - & 1 & 10 & 15 & $25-100$ \\
\hline Construction & - & - & $\mathbf{1}$ & $\mathbf{1 0}$ & $\mathbf{1 0}-\mathbf{2 5}$ \\
\hline
\end{tabular}

Static analysis does not depend on the compiler used and the platform in which the compiled program is executed, thus making it possible to find hidden errors, such as undefined behavioral errors, that may occur even a few years after it was created or errors that may occur in different compilers and platforms. In addition, typos and other errors caused by Copy-Paste usage can be easily and quickly detected.

Static code analysis tools perform these operations according to the rules and standards of the programming language. There are a lot of commercial and free static code analysers. In a research, PMD was deemed to be the best static analysis tool for Java programming language (Abdallah \& Al-Rifaee, 2017).

While most static analysis tools for Java programming langue, use SUN or Google standards, some tools such as PMD use developers approved rules in addition to SUN and Google standards. This makes PMD a multi-standard based tool to implement. In addition, PMD enables the development of tools through the APIs it provides. Therefore, PMD is used in this study.

\subsection{How PMD works?}

PMD is an open source, static code analysis tool with comprehensive configurable rule sets (Thomas et al., 2003). PMD supports Java, JavaScript, Salesforce.com Apex and Visualforce, PLSQL, Apache Velocity, XML, XSL languages (Nembhard et al., 2017).

In PMD, multiple rules or rulesets can be used together, or a custom ruleset can be created. For Java Programming Language, there are more than 280 rules which are defined in eight rulesets: Best Practices, Code Style, Design, Documentation, Error Prone, Multithreading, Performance and Security (PMD n.d.). Additionally, PMD users can execute custom analyses by developing new evaluative rules.

Instead of the source code itself, PMD uses abstract syntax trees (ASTs) created by a JavaCC parser from Java sources. The main loop of PMD then examines AST, visiting all registered rules related to specific AST structures. The rule scan then checks AST and report violations (Aderhold \& Kochtchi, 2013). The following example, given in Figure 3., illustrates rule creating process for PMD and also illustrates inner workings of PMD.

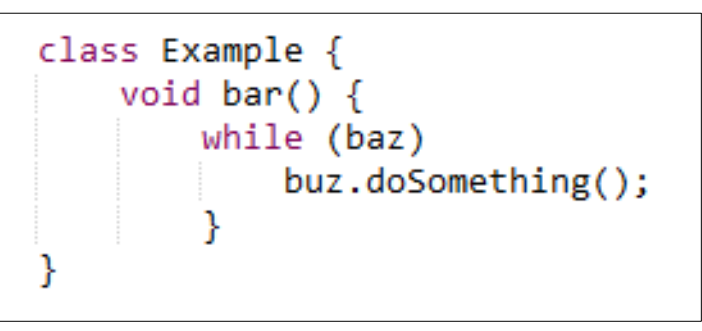

Figure 3. Sample Java code

In PMD, rules are written as Java classes or XPath expression. It actually gets quite difficult to follow source code especially as it gets longer. This is mainly because it is difficult to tell where the curly braces belong. To be able to do this, it is necessary to determine the changes that happen in AST if "buz.doSomething()" clause had braces inserted as shown in Figure 4. 


\begin{tabular}{|c|c|}
\hline $\begin{array}{l}\text { class Example }\{ \\
\text { void bar() }\{ \\
\text { while (baz) } \\
\text { buz.dosomething(); } \\
\} \text { \} }\end{array}$ & $\begin{array}{l}\text { WhileStatement } \\
\text { Expression } \\
\text { Statement } \\
\text { - StatementExpression }\end{array}$ \\
\hline \multicolumn{2}{|c|}{ Code without curly braces } \\
\hline 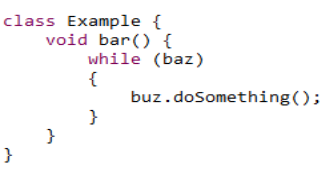 & $\begin{array}{l}\text { WhileStatement } \\
\text { Expression } \\
\text { Statement } \\
\text { Block } \\
\text { BlockStatement } \\
\text { Statement } \\
\text { StatementExpression }\end{array}$ \\
\hline
\end{tabular}

Figure 4. Different AST for sample code with-without curly braces
When the curly braces are added, AST nodes are formed with the names "Block" and "BlockStatement". A rule violation can be detected by writing a rule that detects a "WhileStatement" declaration that is not followed by "Block". This can be done with one of Java class or XPATH experission rule writing methods. To write a custom rule, a new java class needs to be created that is inherited from net.sourceforge.pmd.lang.java.rule.AbstractJavaRule. PMD works by creating AST and then traverses it recursively. By doing this, a rule can get a call back for any type it's interested in. The rule that gets called whenever AST traversal finds a "WhileStatement" can be seen in Figure 5.

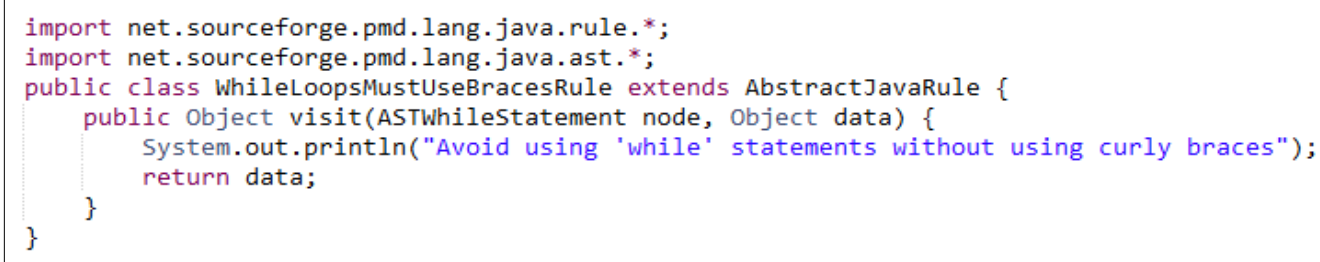

Figure 5. WhileLoopsMustUseBracesRule java code

Once the rule class is written, PMD must be told of this. PMD needs a ruleset XML file to recognize the rule. "sampleCustomRule.xml" file can be seen in Figure 6. The elements and attributes of the file are explained below.

-name - The rule's name.

-message - Message for report.

-class - Location of the rule class.

-description - A description of what this rule looks for. -priority - There are five levels of priority in the PMD for the rules:

1. High priority. Code revision absolutely required.

2. Medium high priority. Code revision highly recommended.

3. Medium priority. Code revision recommended.

4. Medium low priority. Code revision optional.

5. Low priority. Code revision highly optional. - example - A code fragment between CDATA tags to explain the rule violation.

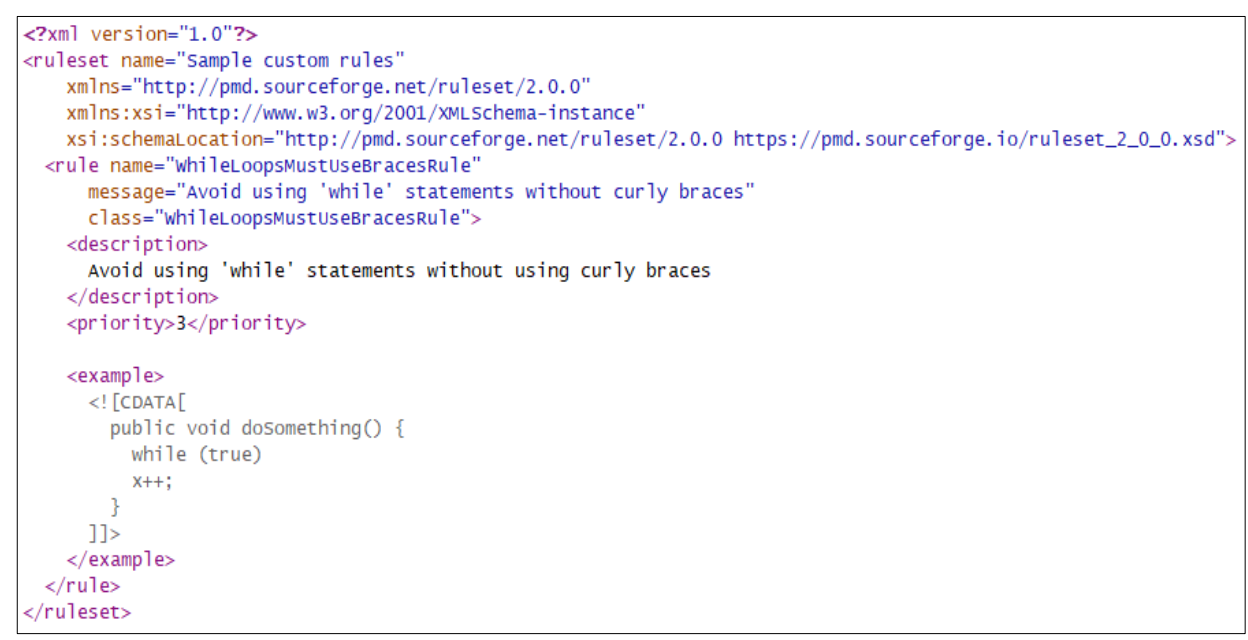

Figure 6. SampleCustomRule.xml

To test the rule run PMD in command line by giving command:

pmd.bat -d "Path to Sample Code" -R "Path to sampleCustomRule.xml"
All findings obtained should be explained with figures and / or charts and avoided from unnecessary repetitions.

After running the command PMD prints "Avoid using 'while' statements without using curly braces", as shown Figure7. 


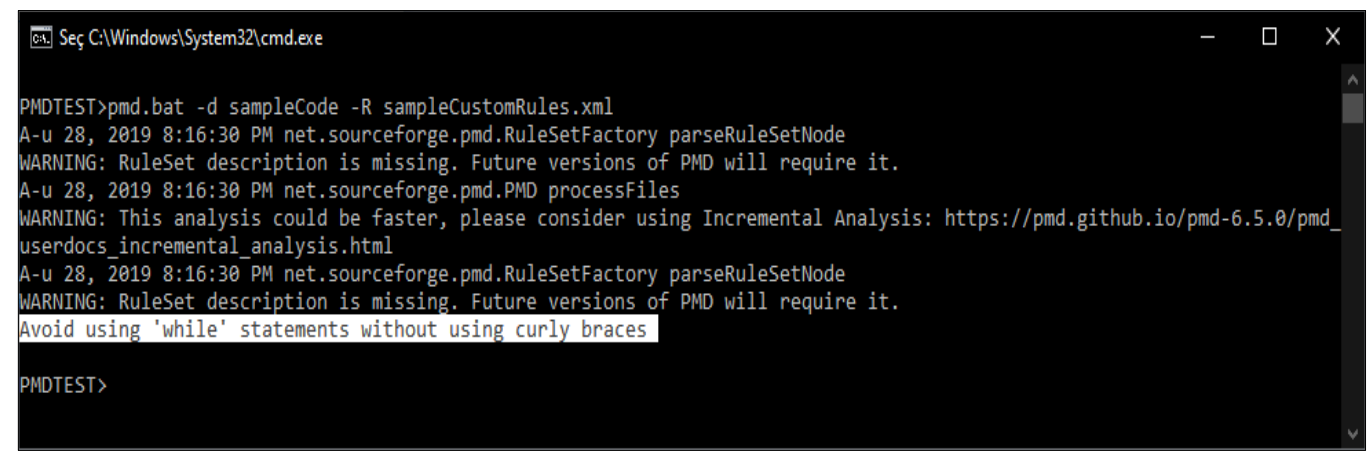

Figure 7. Output of running PMD with sample custom rule

After testing the rule, it is necessary to make changes to the rule class in order to include the rule in the reports to be prepared by PMD. However, since the aim is to explain how PMD works instead of preparing a custom rule for PMD, the continuation of the subject and/or preparing the rule with XPATH expressions will not be included here.

\subsection{Operating PMD}

PMD is distributed as a zip archive. The latest binary distribution can be downloaded from the releases page. In Windows operating system, to run PMD, unzip it in any directory and run the file "pmd.bat" under the "bin" folder with the required parameters from the command line. PMD does not have a graphical user interface. pmd.bat requires two arguments:

-d <path>: Path to files of source code to analyse.

- $\mathrm{R}<$ path>: The ruleset file. PMD uses xml configuration files.

Other arguments of PMD are optional. For instance, PMD displays the report on command line by default. But user can change this by giving "-r" argument with a path to a file in which the report output will be recorded. Full list of arguments can be found on PMD's documentation page.

\section{RESULTS}

\subsection{Shortcomings of PMD and Java Project Analyser (JPA)}

Although the generated report will be displayed on the command screen or stored in the name and type specified at each run, PMD will not operate if the parameters are missing or incorrect. Moreover, it will overwrite a previous existing report if the same name is given as a parameter again without warning. Another major difficulty with PMD is keeping track of the names of the rulesets. Most importantly it will not be able to compare the report files. Java Project Analyser (JPA) was developed to prevent these hurdles and improve the user experience in this study. Using JPA is considerably easier because it can be used via the graphical user interface after running the file named JPA.jar.

\subsection{How JPA works}

The following describes how JPA works with sample test codes. JPA is developed for this study and its main window can be seen in Figure 8 .

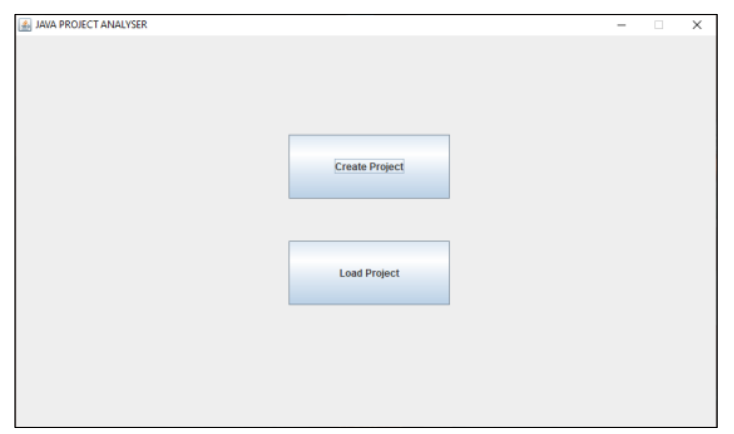

Figure 8. JPA main window

As shown in Figure 8, when JPA is first run, a window with two buttons is displayed. The first button can be used to create a new project for analysis, or the second button can be used to re-analyze an existing project or compare old analyses. To test with JPA, two source code files named "CodingHorror.java" and "StringHorror.java" are prepared in the "TestCodeFolder" folder and the codes are given in Figure9. 


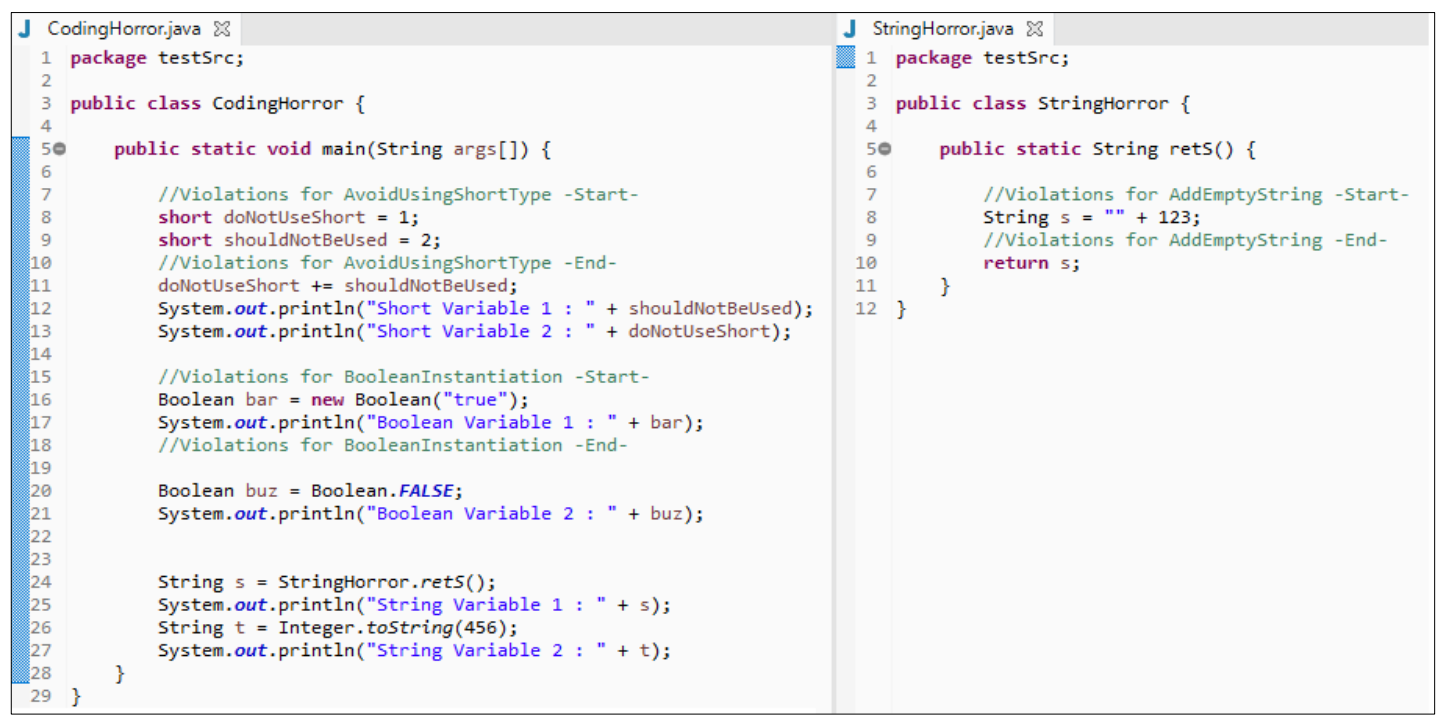

Figure 9. CodingHorror.java and StringHorror.java

These codes run successfully and are sent to the command screen. However, there are three violations in these codes, one high priority (1), one medium high priority (2) and one medium priority (3) in the performance ruleset of PMD. Explanations for these three violations are given in Figure 10 .

\begin{tabular}{|l|c|l|}
\hline \multicolumn{1}{|c|}{ Rule Name } & Priority & \multicolumn{1}{c|}{ Definition } \\
\hline AvoidUsing ShortType & 1. High & $\begin{array}{l}\text { Using "short" data type is beneficial for memory. However, } \\
\text { since JVM can only perform arithmetic operations for data } \\
\text { types "int" and "long", it requires to convert "short" to "int" } \\
\text { when processing the value, and converts the result back to } \\
\text { "short". This would result for losing of performance when } \\
\text { trying to get more memory gains. }\end{array}$ \\
\hline BooleanIn stantiation & $\begin{array}{c}2 . \\
\text { Medium } \\
\text { High }\end{array}$ & $\begin{array}{l}\text { Avoid using "new Boolean()" object. It can be referenced } \\
\text { "Boolean.TRUE" or "Boolean.FALSE" instead. Also, using } \\
\text { "new Boolean()" object is deprecated since JDK 9. }\end{array}$ \\
\hline AddEmptyString & $\begin{array}{c}3 . \\
\text { Medium }\end{array}$ & $\begin{array}{l}\text { It is better to use one of the type-specific "toString0" methods } \\
\text { to convert variable types to "string" instead of aggregating } \\
\text { them with empty string ("). }\end{array}$ \\
\hline
\end{tabular}

Figure 10. PMD violations in sample codes

To analyze the test code with JPA, select the folder with the "Create Project" button as in Figure 11.

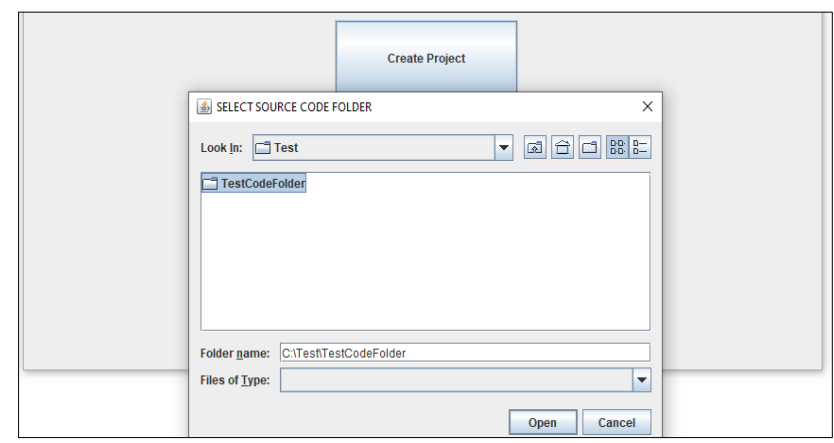

Figure 11. Selecting source code folder

After selecting the folder, ruleset(s) need to be selected to run analysis otherwise program will give error as shown Figure 12.

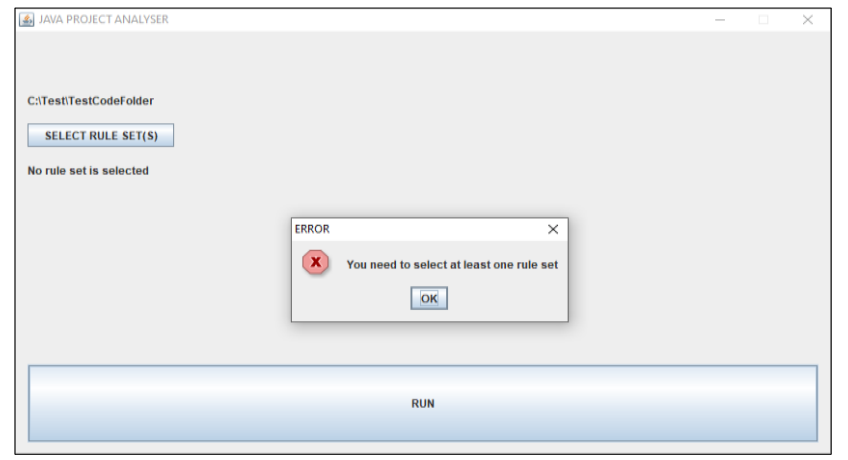

Figure 12. Error because ruleset(s) is not selected

"SELECT RULE SET(S)" button is used to select the ruleset(s). From the window that opens, the rulesets can be selected. For the test code "Performance" rule set was used, as in Figure 13.

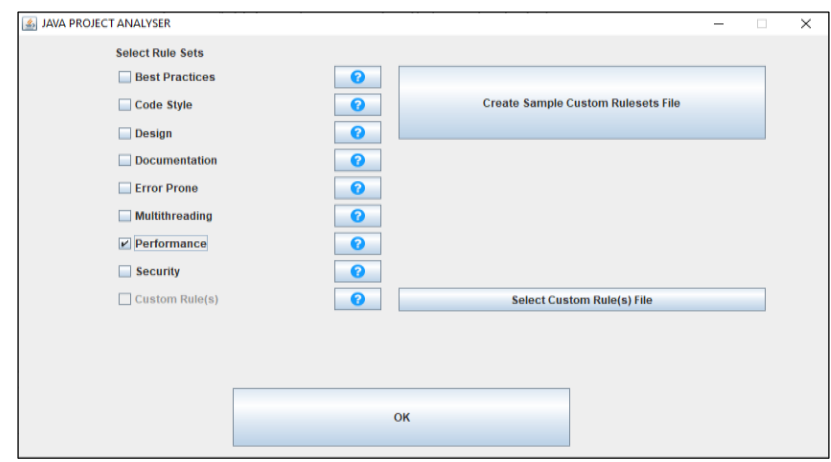

Figure 13. Ruleset(s) selection

Considering that this was just a demonstration and not a complete test, it was not necessary to use the entire performance ruleset for the test codes here. Since the example codes are known to contain three violations, "Custom Ruleset" file could be prepared in "xml" format as shown in Figure 14. and used at the rule selection shown in Figure 13. 


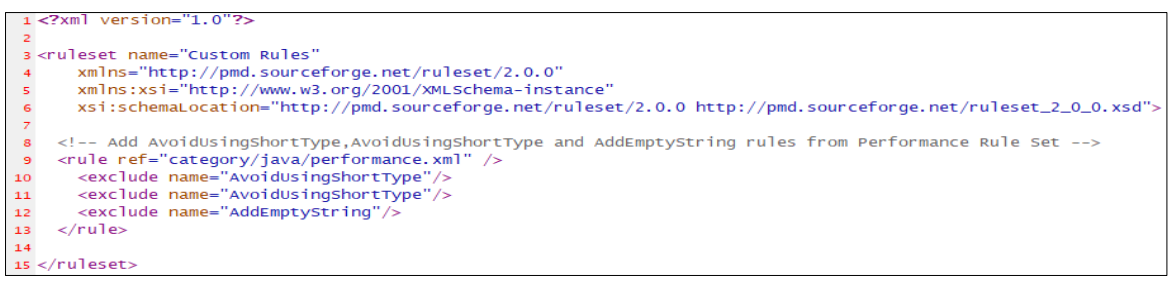

Figure 14. Custom ruleset $\mathrm{xml}$ file

After returning with "OK" button, selected rule sets are listed and with "RUN" button analysis starts. JPA uses PMD APIs to analyze the project and stores the generated report in SQLite database with the name ".AOP.db" in the same folder as test codes. To open the report, press the "OPEN REPORT" button as shown in Figure 15. The schema of the database is given in Figure 16.

As can be seen in Figure 17., the report can be reviewed together for all source code files or separately for each source code file.

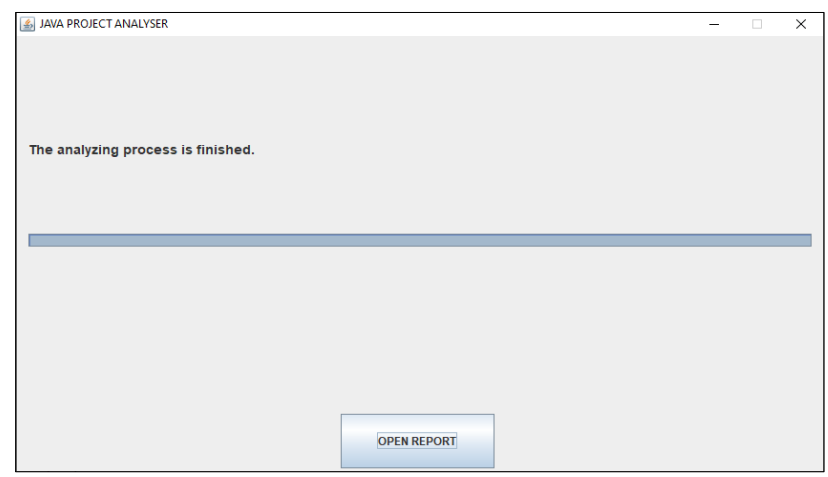

Figure 15. Post analysis confirmation screen

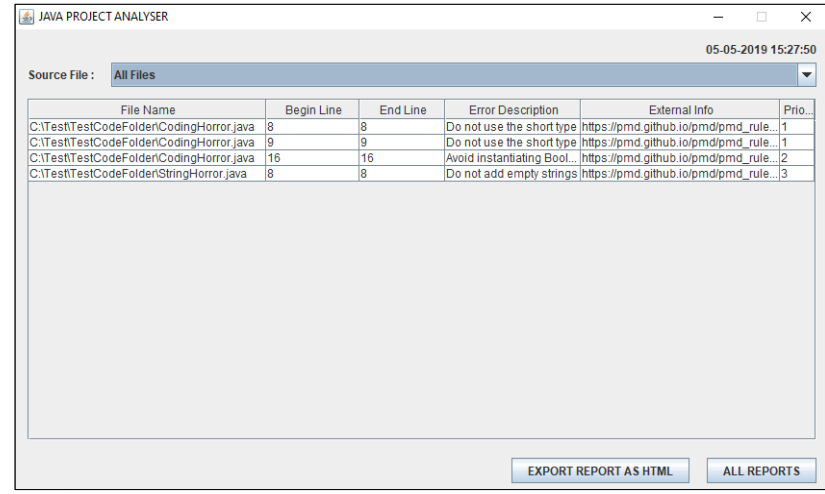

Figure 17. Generated report

"ALL REPORTS" button opens the window listing all the reports. In this window, the old reports can be viewed, the reports can be deleted or compared. At least two reports are needed for comparing or program gives error as expected shown in Figure 18.

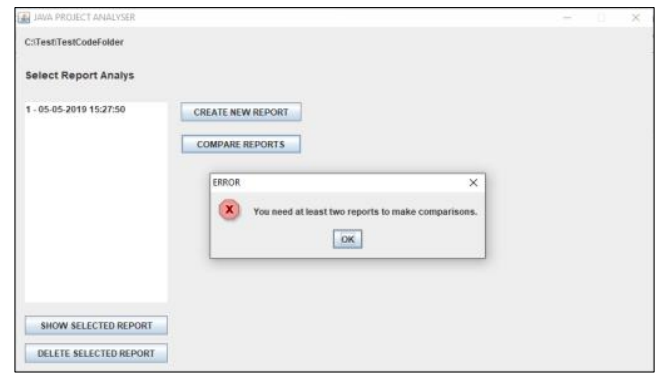

Figure 18. Error when try to compare reports

\begin{tabular}{|c|c|c|c|c|c|}
\hline \multicolumn{2}{|l|}{ report } & \multicolumn{2}{|l|}{ file } & \multicolumn{2}{|l|}{ violation } \\
\hline \multirow{14}{*}{$\begin{array}{l}\text { report_Id } \\
\text { pmd_version } \\
\text { timestamp } \\
\text { totalViolationCount }\end{array}$} & integer & file_id & integer & violation_id & integer \\
\hline & text & report_id & integer & file_id & integer \\
\hline & text & name & text & beginline & text \\
\hline & text & & & endline & text \\
\hline & & configer & & begincolumn & integer \\
\hline & & ce_id & integer & endcolumn & integer \\
\hline & & report_id & integer, & rule & text \\
\hline & & rule & text & ruleset & text \\
\hline & & & & package & text \\
\hline & & & & class & text \\
\hline & & & & method & text \\
\hline & & & & externallnfoUrl & text \\
\hline & & & & priority & integer \\
\hline & & & & content & text \\
\hline
\end{tabular}

Figure 16. Database scheme for ".AOP.db" 
Before creating new report, change the codes like in Figure 19. to eliminate violations. In order to ignore a violation and keep the code by PMD, just type "//NOPMD" at the end of that line if the violation consists of a single line. For multi-line violations there are several ways to ignore can be found on PMD documentation under "Suppressing Warnings" title.
In this example a single line suppression was typed in line 8 of the "StringHorror.java" source file, "short" variable type in lines 8 and 9 has been replaced with "int" in the source file "CodingHorror.java". Only the violation in line 16 remains.

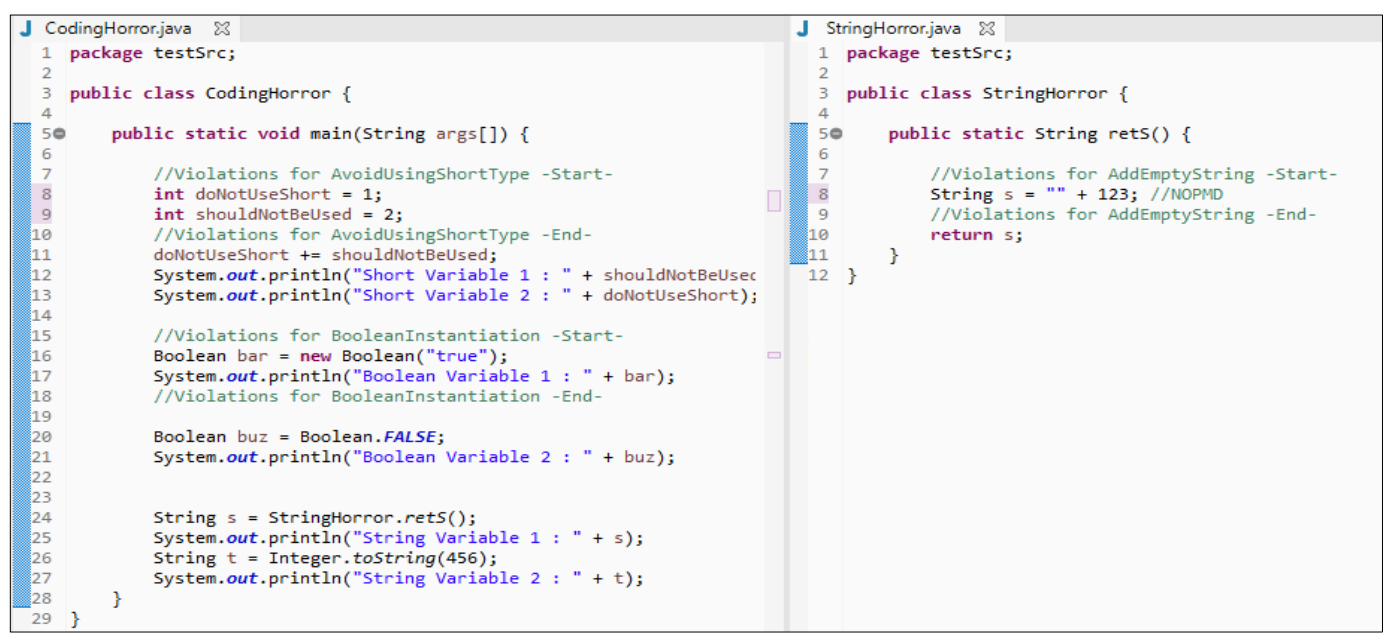

Figure 19. Updated CodingHorror.java and StringHorror.java source code files

Figure 20 shows that only the "BooleanInstantiation" violation is reported when the analysis is performed again.

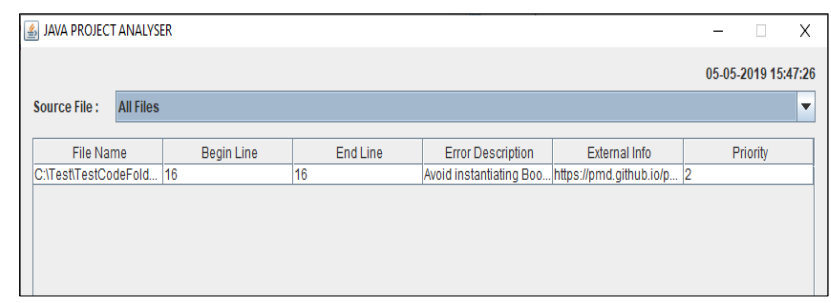

Figure 20. Generated report for updated codes

When comparing the two reports, as in Figure 21., only this violation is common.

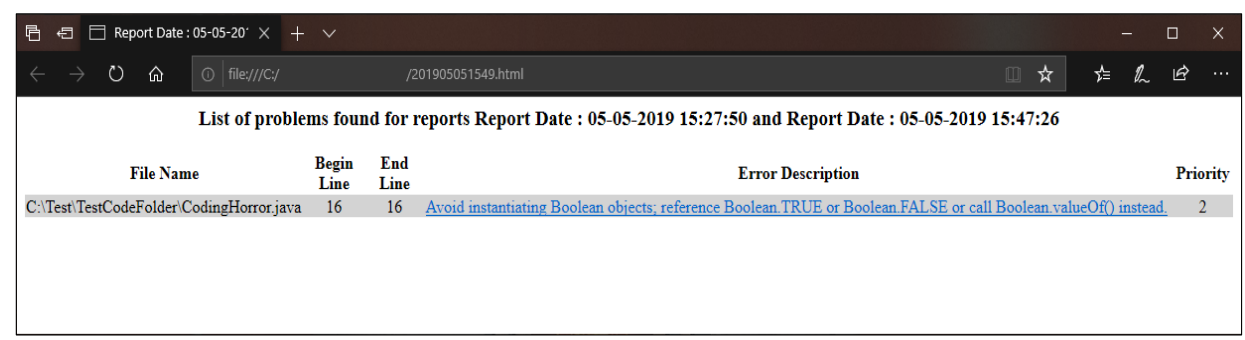

Figure 22. Comparison of reports exported as html
When reviewing reports in JPA, documentation page of a violation can be accessed from the column named "External Info". The same applies to the "Error Description" column when exporting the report as "html". The image of a sample

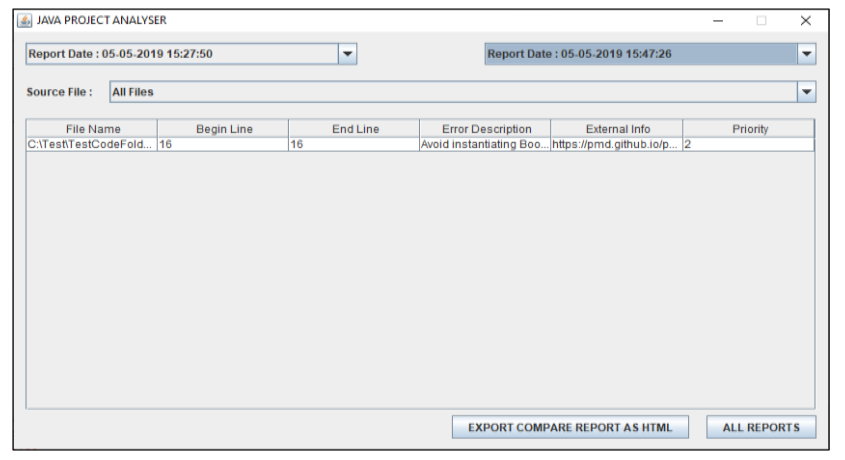

Figure 21. Comparison of reports

As in a single report view, the comparison of two reports can be exported as "html". Figure 22. shows the comparison of reports exported as "html". documentation page for "BooleanInstantiation" violation that can be accessed through the link in the report in Figure
22 . can be seen in Figure 23. 


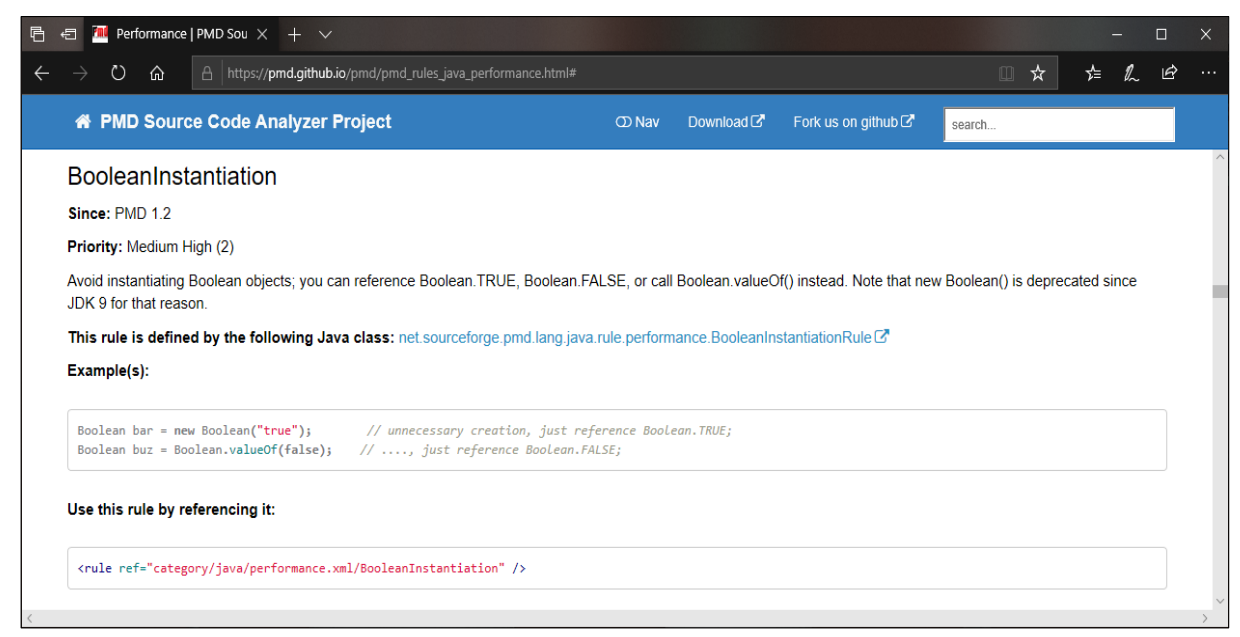

Figure 23. Documentation page for "BooleanInstantiation" violation

If a previously analyzed folder is selected as destination while creating a new project, it will notify user of the issue and ask whether to load the existing project or not, as shown in Figure 24. Similarly selecting an un-analyzed folder while loading a project will bring an appropriate notification, asking the user to create a new project or not as in Figure 25.

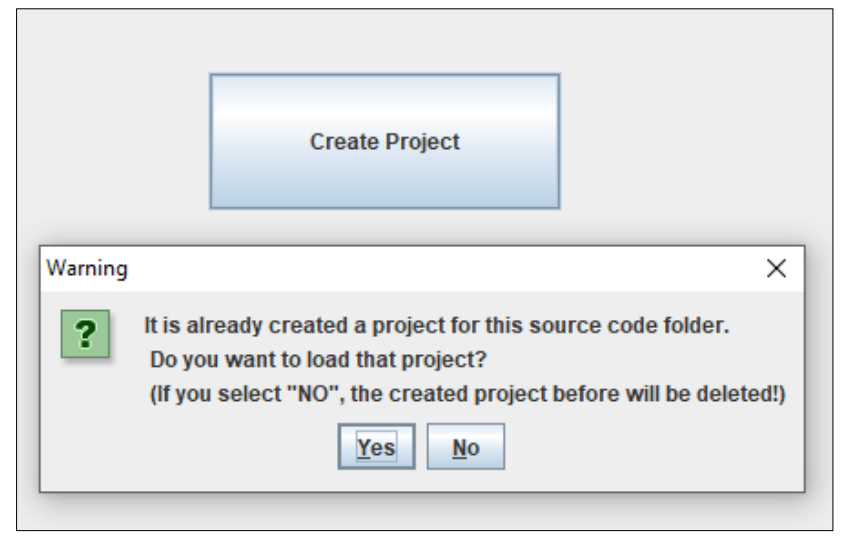

Figure 24. Warning for an already existing project where the new project is wanted to be created

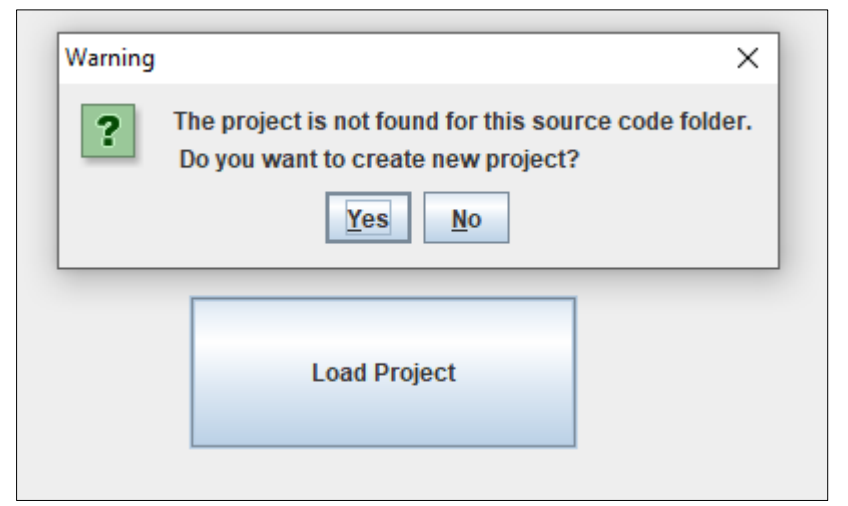

Figure 25. Warning for no existing project where the project is wanted to be loaded

\section{DISCUSSION AND CONCLUSIONS}

In this study, a platform independent tool is designed to perform static code analysis. The speed of execution of Java code is highly dynamic and fundamentally depends on Java Virtual Machine. An old piece of Java code may well

run faster on a more recent JVM, even without recompiling Java source code. Combining this fact with the possibility that refactoring may not sufficiently improve the software performance, software testing approach was preferred. It was decided to implement white box approach at source code level with statement coverage to optimize code. In order to cover all code lines, a tool has been developed based on PMD source code analyzer to perform static code analysis automatically and compare the analysis outputs.

Although PMD itself was a good starting point for static analysis there were obvious shortcomings to be improved. Plugins have been developed to integrate PMD source code analyzer into IDEs, Ant and Maven build tools by third parties. JPA, the tool built in this study, on the other hand has its own graphical interface and also can store and compare reports in its own database of projects. It has fundamentally been developed to improve the user experience in automating static code analysis. As a standalone program, JPA only needs source code for code analysis and does not require any IDEs or compilers. Since JPA keeps the reports methodically in a database form it can also be a useful tool in software testing development process.

This study provides detailed information about PMD source code analyzer and the tool built on PMD through its API's. Future studies may be done on automatic estimation for deciding which rulesets to use in code analysis using machine learning. Additionally, custom rule creation process may also be studied for improvement in the future. Furthermore, forthcoming updates and developments in PMD may inspire similar studies, or new ideas under new conditions that prevail at the time. 


\section{REFERENCES}

Abdallah, M. M., \& Al-Rifaee, M. M. (2017). Java Standards: A Comparative Study. International Journal of Computer Science and Software Engineering, 6 (6), 146-151.

Aderhold, M., \& Kochtchi, A. (2013). Tailoring pmd to secure coding. Tech. Rep.

Ayewah, N., Pugh, W., Hovemeyer, D., Morgenthaler, J. D., \& Penix, J. (2008). Using static analysis to find bugs. IEEE Software, 25 (5), 22-29.

Bajwa, M. S., Agarwal, A. P., Gupta, N. (2016) Code optimization as a tool for testing software. 3rd International Conference on Computing for Sustainable Global Development, 961-967.

Carpenter, B., Chang, Y. J., Fox, G., Leskiw, D., \& Li, X. (1997). Experiments with 'HP Java'. Concurrency: Practice and Experience, 9(6), 633-648.

Galin, D. (2004). Software quality assurance: from theory to implementation. India: Pearson Education.

Gosling, J., Joy, B., Steele, G., Bracha, G., Buckley, A., Smith, D. (2018) The Java(TM) Language Specification Java SE 11 Edition, Retrieved November 14, 2018, from https://docs.oracle.com/javase/specs/

Hall, S. P. \& Anderson, E. (2009) Operating systems for mobile computing. Journal of Computing Sciences in Colleges, 25 (2), 64-71.

Jovanović, I. (2009) Software testing methods and techniques. The IPSI BgD Transactions on Internet Research, 5 (1), 30-41.

Johnson M. (2008) Code Optimization. Handout 20.

Karnavel, K., \& Santhoshkumar, J. (2013, February). Automated software testing for application maintenance by using bee colony optimization algorithms (BCO). In 2013 International Conference on Information Communication and Embedded Systems (ICICES), 327-330

Khan, M. E., \& Khan, F. (2012). A comparative study of white box, black box and grey box testing techniques. International Journal of Advanced Computer Science and Applications, 3 (6), 12-15.

Knuth, D. E. (1974). Computer programming as an art. Communications of the ACM, 17(12), 667-673.

Kotlin (n.d.). Retrieved January 5, 2019, from https://kotlinlang.org

Lins, F. M. (2017) The effects of the compiler optimizations in embedded processors reliability. MSc Thesis, Universidade Federal Do Rio Grande Do Sul, Porto Alegre

Moreira, J. E., Midkiff, S. P., Gupta, M., Artigas, P., Wu, P., \& Almasi, G. (2001). The ninja project: Making java work for high performance numerical computing. Commun. ACM, 44(10), 102-109.
McConnell, S. (2004). Code complete (2nd ed.). Redmond, Washington: Microsoft Press.

Merriam-Webster (n.d.). Retrieved November 15, 2018, from https://www.merriamwebster.com/dictionary/optimization

Nembhard, F., Carvalho, M., \& Eskridge, T. (2017). A hybrid approach to improving program security. In 2017 IEEE Symposium Series on Computational Intelligence (SSCI) 1-8.

Palaniappan S. (2016) Recent trends and challenges in source code optimization. International Journal of Trend in Research and Development, 3(6), 603-607.

PMD (n.d.). Retrieved April 3, 2019, from https://pmd.github.io/pmd6.5.0/pmd_rules_java.html

Sawant, A. A., Bari, P. H., Chawan, P. M. (2012). Software testing techniques and strategies. International Journal of Engineering Research and Applications (IJERA), 2 (3), 980-986.

Scala (n.d.). Retrieved January 5, 2019, from https://www.scala-lang.org

Singh, A. H., \& Kazi, N. N. (2016) Software Testing Mumbai: Himalaya Publishing House Pvt. Ltd.

Statcounter (n.d.). Retrieved November 15, 2018, from http://gs.statcounter.com/os-market-share

Watson, M. (2017). Why Premature Optimization Is the Root of All Evil. Retrieved January 3, 2019, from https://stackify.com/premature-optimization-evil/ 